\title{
A New 3D Model Retrieval Method with Building Blocks
}

\author{
Mingquan Zhou, ${ }^{1}$ Qingsong Huo, ${ }^{2}$ Guohua Geng, ${ }^{2}$ and Xiaojing Liu ${ }^{3}$ \\ ${ }^{1}$ College of Information Science and Technology, Beijing Normal University, Beijing 100875, China \\ ${ }^{2}$ College of Information Science and Technology, Northwest University, Xi'an 710127, China \\ ${ }^{3}$ Department of Computer Technology and Application, Qinghai University, Xining 810016, China
}

Correspondence should be addressed to Qingsong Huo, huoqingsong@126.com

Received 31 January 2009; Accepted 19 February 2009

Recommended by Suiping Zhou

\begin{abstract}
As the numbers of 3D models available grow in many application fields, there is an increasing need for a search method to help people find them. Unfortunately, traditional search techniques are not always effective for 3D data. In this paper, we describe a novel method of interactive 3D model retrieval with building blocks. First, by using a cube block as the baseblock in a 3D virtual space, we may construct the query model with human-computer interaction method. Then through retrieving the polygon model of the database generated by the voxel model, we may get retrieval results in real time. Experiments are conducted to evaluate the performance of the proposed method.
\end{abstract}

Copyright (C) 2009 Mingquan Zhou et al. This is an open access article distributed under the Creative Commons Attribution License, which permits unrestricted use, distribution, and reproduction in any medium, provided the original work is properly cited.

\section{Introduction}

With the developments of computer graphics and the progress in multimedia hardware technologies, 3D models are emerging in many application fields such as game, medicine, and molecular biology and are playing more important role in multimedia data types. 3D model has gradually become the fourth multimedia data type after voice, image, and video. Consequently, achieving effective and efficient content-based 3D model retrieval has now become a hotspot in the research of multimedia information retrieval [1]. How to search useful and the same theme of the models usefully and effectively has become the main goal of 3D model retrieval. In order to provide users with a convenient way of search, a mature retrieval system should have a good interactive performance.

Compared with images and other 2D media, 3D models contain more rich information. Therefore, 3D model of content-based retrieval system generally has a variety of query approaches $[2,3]$. Some ways to retrieve of a desired model from a large selection of 3D shape models have already been proposed, for example, the text-based search method, 2D hand-drawn draft search method, 3D hand-drawn draft search method, the example of 3D models search method, and interactive retrieval of 3D shape models using physical objects [4-9].
Currently, the text-based search method is popular. It only needs to enter the keyword, but a text description is often too limited and incorrect. The most important thing is that we must artificially mark models firstly, and it is not realistic to the mass of the models data. $2 \mathrm{D}$ hand-drawn draft search method is to produce an effective $2 \mathrm{D}$ visual draft for retrieval, and Princeton University presents a draft manual mapping 2D retrieval interface [5]. Pu and Ramani [10] retrieve by drawing images. Cao et al. [11] and Fonseca et al. [12] retrieve by drawing $2 \mathrm{D}$ sketch according to the query model provided by the users. Although users can express their intentions by drawing 2D sketch, it improves the users' requirements. It is very difficult to transform $3 \mathrm{D}$ models into $2 \mathrm{D}$ images especially for the users who are beginners of studying computer graphics and computer-aided design. In order to input 3D models, Igarashi et al. [13] design a 3D sketching tool Teddy. Hou and Ramani [14] draw 3D part models and retrieve by cluster rules. Compared to the draft drawn 2D sketch, it has more difficulty and more restrictions for drawing 3D sketch. Moreover, the 3D sketching tool is, arguably, hard to use especially for a person who does not have a talent for painting or drawing. Examples of 3D models require users to retrieve the first sample to provide a $3 \mathrm{D}$ model. Users can easily operate, but without a suitable 3D model as the retrieval example, it is very difficult to carry on. 


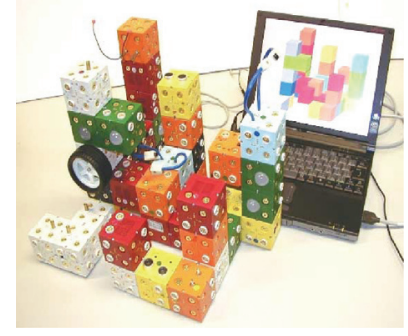

(a)

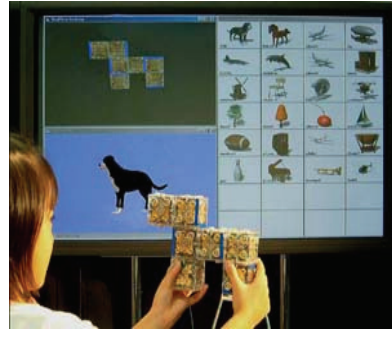

(b)
FIgURE 1: Appearance of the ActiveCube system.

Siegl et al. [15] employ an interactive approach to teaching and retrieving by using mobile AR-kits. Ichida et al. [16] implement a query interface for retrieval of 3D shape models with physical objects by using the ActiveCube system. The appearance of the ActiveCube system is shown in Figure 1.

The query model of interactive retrieval of 3D shape models using physical objects is constructed by the users themselves, without converting into images or 3D drawing sketch. The retrieval interface is very simple, and users may participate in the query process. But the retrieval method must use physical objects as the media; otherwise, it is unable to carry on. Furthermore, it is restricted by only six surfaces of the connection and the number of physical objects. The expression of models is very weak. Is there a better retrieval way? This paper proposes a new 3D model retrieval method with building blocks.

The rest of the paper is organized as follows: 3D model retrieval method with building blocks is introduced in Section 2. Section 3 gives experimental results to show the effectiveness with the proposed search method. Section 4 presents the method of retrieval optimization. Finally, conclusions are given in Section 5.

\section{3D Model Retrieval Method with Building Blocks}

Building blocks is a common Children's toy. By a few simple building blocks, it combines various characters, animals, bridges, houses, towers, and so on. This paper is inspired by the building blocks in the game and structures a virtual environment in the computer. It constructs 3D query models by building blocks, expresses the users' expression, and realizes 3D models retrieval.

2.1. Retrieval Method Introduction. As shown in Figure 2, the retrieval system sample points to $3 \mathrm{D}$ polygon models and builds voxel model database. Then users construct the query online model. Finally the system makes similarity computations and gets the query result by contrasting the query model with the models of voxel model database.

2.2. Generating Voxel Model. We assume that the entire 3D model is composed by the triangle mesh $T=\left(T_{1}, T_{2}, \ldots, T_{n}\right)$.
$A, B, C$ are the vertices. The vertex density or mesh density varies greatly. In order to effectively describe the characteristics of 3D models, this paper samples points by subdividing triangle mesh.

As shown in Figure 3 , the triangle mesh $(A, B, C)$ is to be subdivided, $(B, C)$ is the longest side, and $\mathrm{O}_{3}$ is the midpoint of $B C$. So the triangle mesh may be subdivided into two triangle meshes, and they are the triangle mesh $\left(A, B, O_{3}\right)$ and the triangle mesh $\left(A, O_{3}, C\right)$. If the area of the triangle meshes $\left(A, B, O_{3}\right)$ and $\left(A, O_{3}, C\right)$ is more than a threshold, we will continue subdividing the triangle meshes $\left(A, B, O_{3}\right)$ and $\left(A, O_{3}, C\right)$ as that of the triangle mesh $(A, B, C)$. Iteration will not stop until the area of the subdivided triangle mesh is less than the threshold $T$. We call the center of the subdivided triangle mesh as the sampling points of the $3 \mathrm{D}$ model. In Figure $3, P_{1}, P_{2}, P_{3}$, and $P_{4}$ are sampling points. And all the sampling points of triangle meshes constitute voxel model of polygon model. Figure 4(a) is a chair model, and Figure 4(b) is a voxel model of chair model.

2.3. Construction of Query Model. This paper uses a cube as the baseblock of building blocks. We construct query model by building blocks with many baseblocks. Construction interface is shown in Figure 5. In the construction interface, each baseblock is expressed by a red cube. The red cube has 6 surfaces, 8 angles, 22 sides, and 26 kinds of connections direction. We may build blocks in each direction, and the yellow translucent cube represents the next baseblock which will be built.

The construction process of query model is as follows.

(1) Click on any red block in the interface, then we will see 26 semitransparent connected yellow baseblocks.

(2) When clicked, the semitransparent baseblock becomes red baseblock.

(3) According to the need of the constructing model, repeat (1), (2) and continue building blocks until we are satisfied.

Through the above process, we can get a satisfactory query model. Figures 6 and 7 are, respectively, the constructing process of a plane model and a stool model.

2.4. Similarity Computations. We suppose that the query model is constructed by $n$ baseblocks according to the prior process, and we describe the details of this similar calculation procedure in the following set of steps.

(1) Bound the query model constructed by $n$ cube-blocks with bounding box, obtain the minimum size of its bounding box, and denote with $M$.

(2) Pick one of polyhedral models, sample it with the method of 2.2, and obtain its voxel model and the minimum size of its bounding box, denoting with $N$.

(3) Assume that the smallest box $M$ is segmented into $a \times b \times c$, and the smallest box $N$ is also segmented into $a \times b \times c$. The segmenting progress is shown in Figure 8 . 


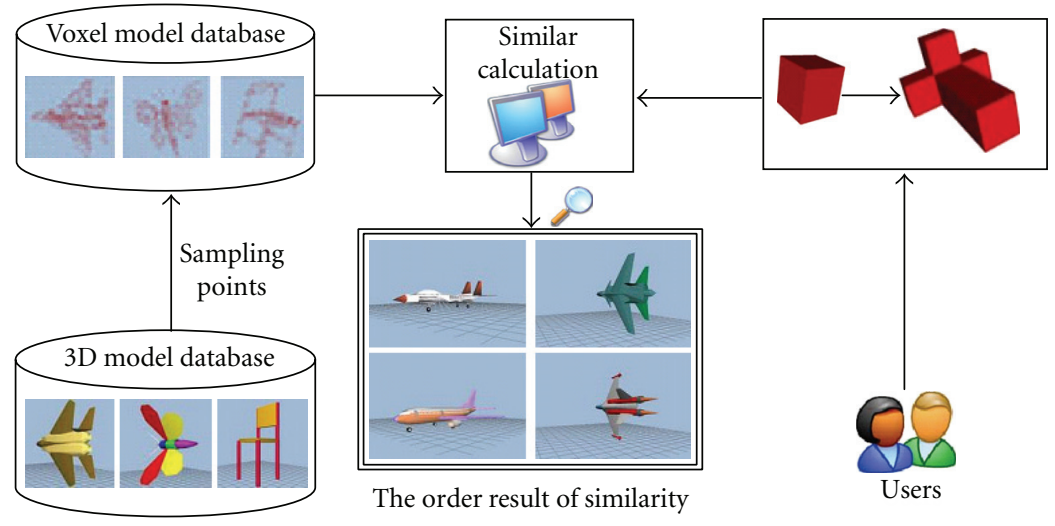

FIGURE 2: System flow chart.

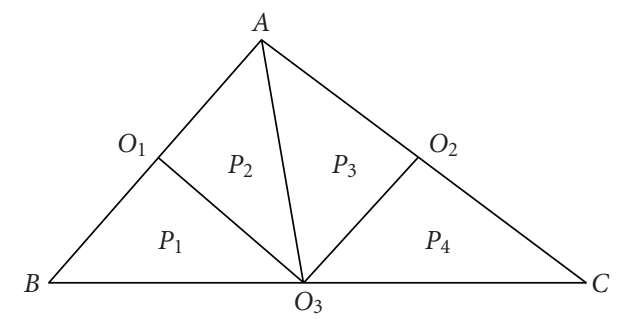

Figure 3: Triangle mesh and sampling points.

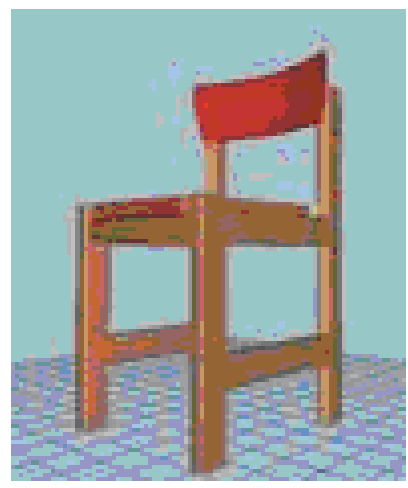

(a)

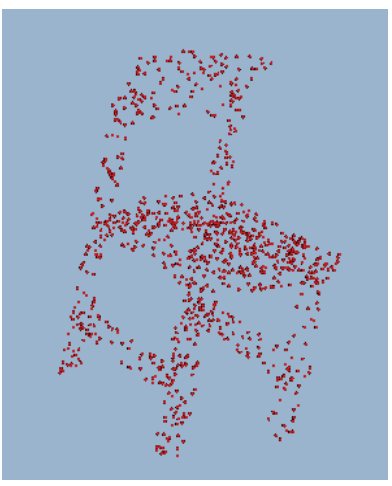

(b)
Figure 4: Model chair and its voxel model.

(4) Assume the number of points of the voxel model is, respectively, $P_{1}, P_{2}, \ldots, P_{n}$ which fall into the baseblock. We compute the total number of points which fall into the query model in accordance with formula (1):

$$
\begin{gathered}
P^{\prime}=\sum_{i=1}^{n} P_{i}, \\
\operatorname{Sim}=\frac{P^{\prime}}{P} \times 100(\%) .
\end{gathered}
$$

(5) Flip the smallest bounding box and repeat (3)-(5) until the six flip manners are all processed. Receive maximum $P^{\prime}$ and make similarity computations in accordance with formula (2).

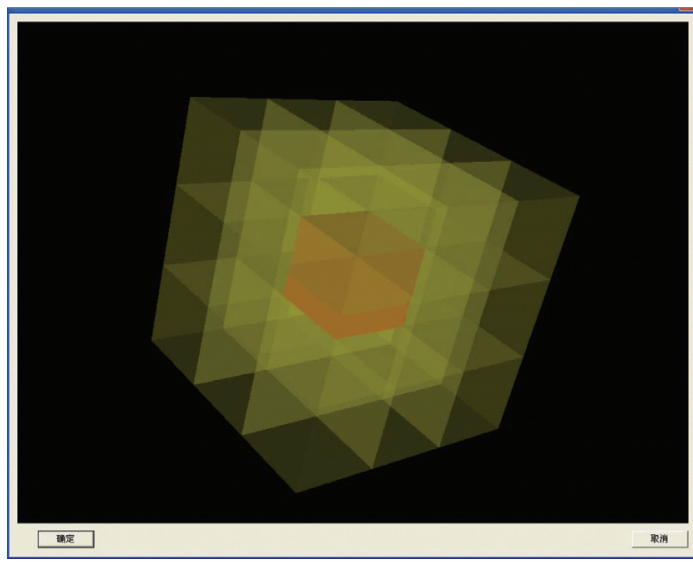

Figure 5: Query model construction interface.

(6) Repeat (2)-(5) operation until the models of the database are all processed.

(7) According to high to low arrangement order, we will be able to get retrieval results.

\section{Experimental Results and Performance Evaluation}

In this paper, the experimental data model is the Princeton University $3 \mathrm{D}$ retrieval database $\mathrm{PSB}$, which contains a total of 1814 models.

3.1. Experimental Results. We construct the plane model and stool as a query model with the method as described above. Their experimental results are shown in Figures 9 and 10.

3.2. Performance Evaluation and Analysis. From Figures 9 and 10 of the retrieval results, we can see that the plane model retrieval result is better than that of the stool model. In order to further test the accuracy of retrieval results, we use recall 


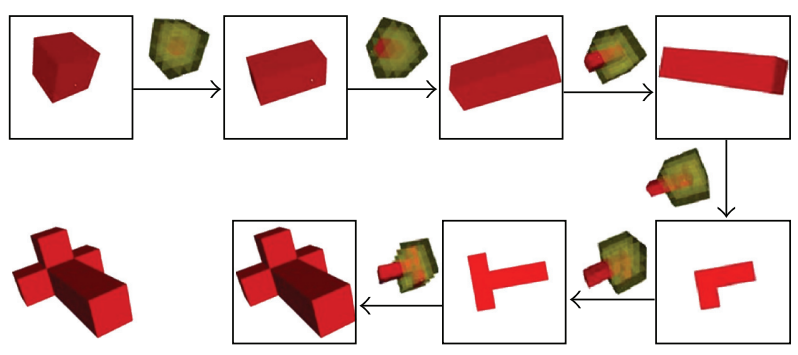

FIGURE 6: The constructing process of a plane model.

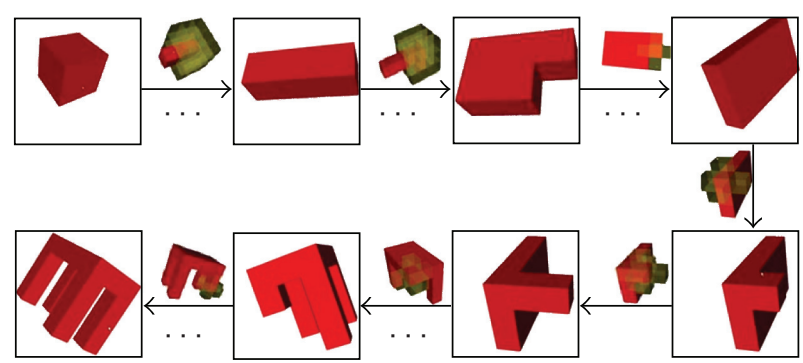

FIgURE 7: The constructing process of a stool model.

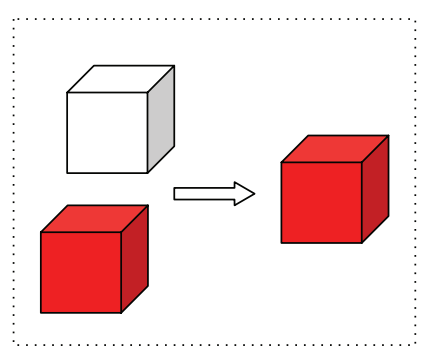

(a)

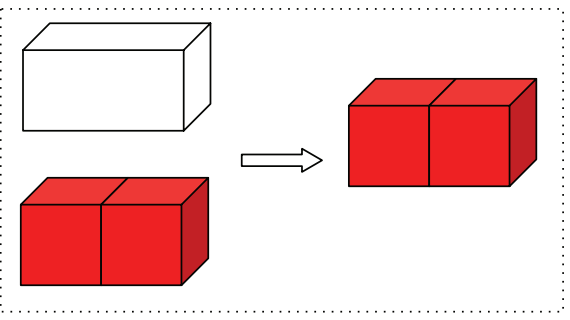

(b)

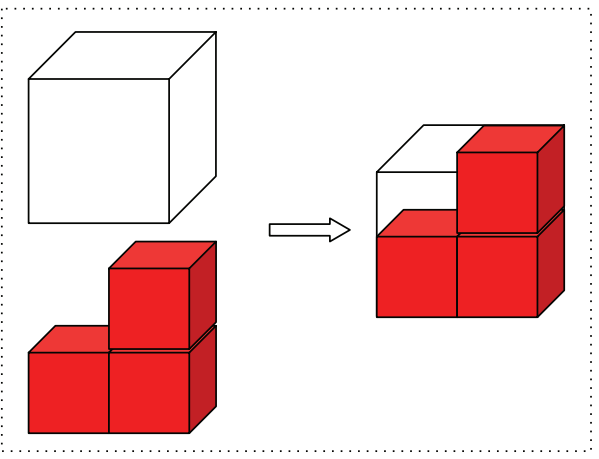

(c)

FIgURe 8: Model of the split-sample map.

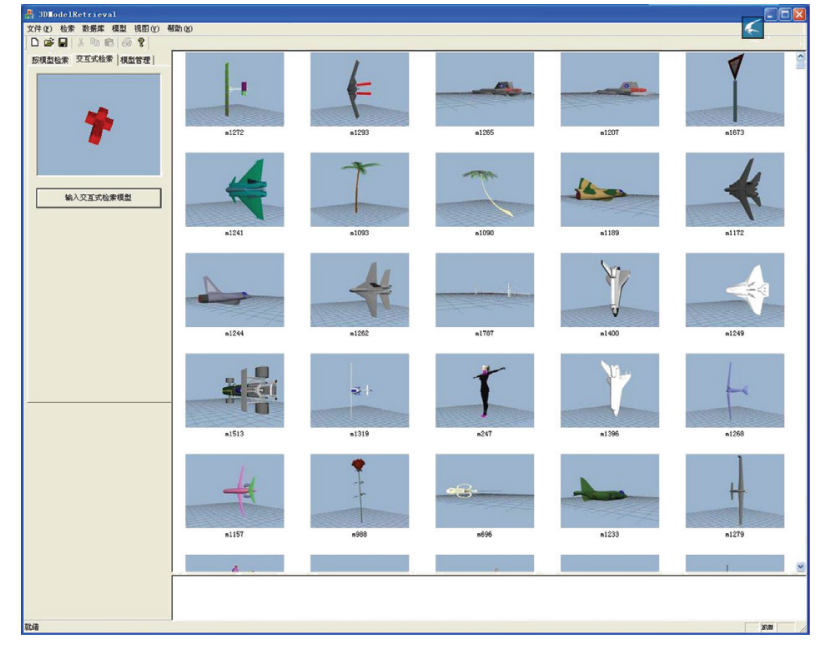

Figure 9: The plane model experimental result.

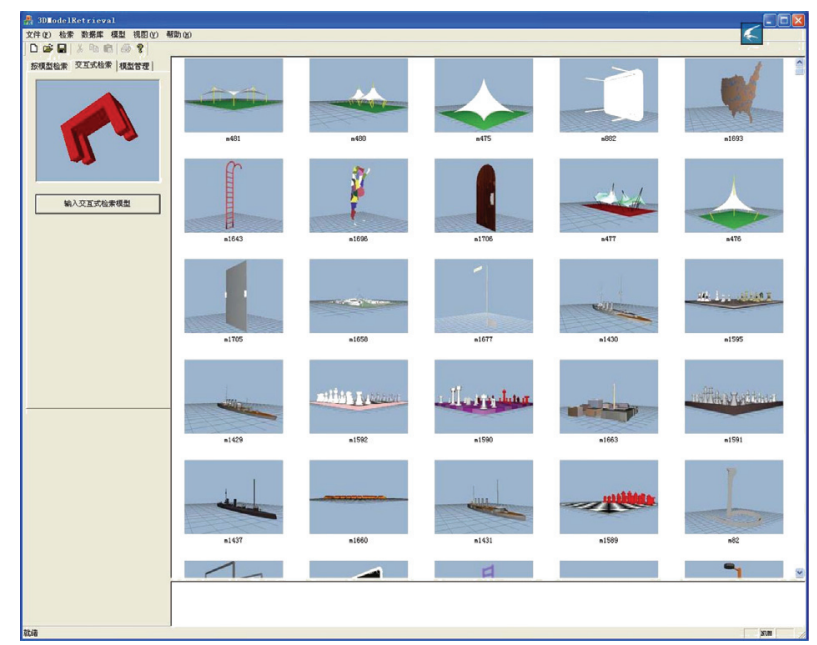

Figure 10: The stool model experimental result.

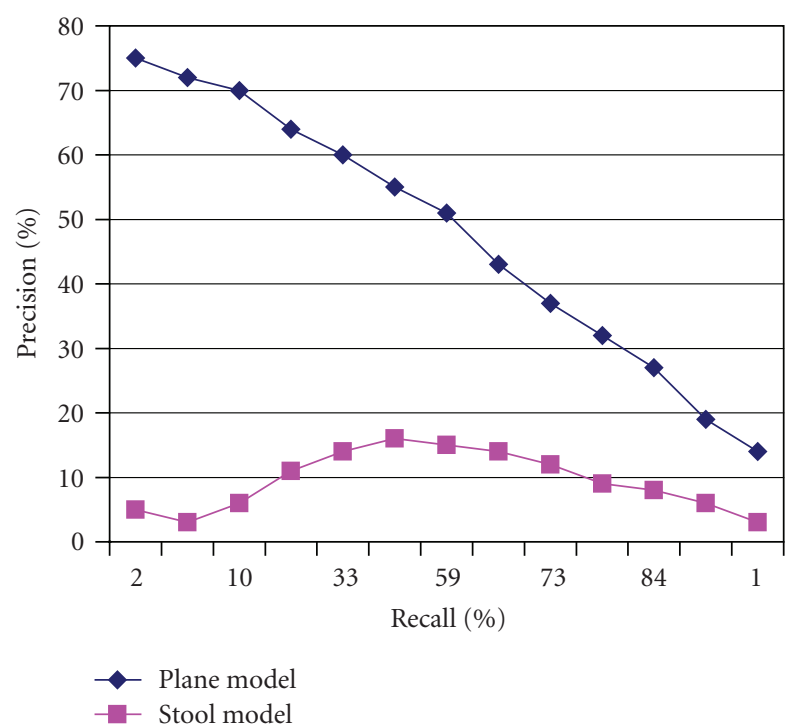

Figure 11: Precision recall of the plane and the stool models. 


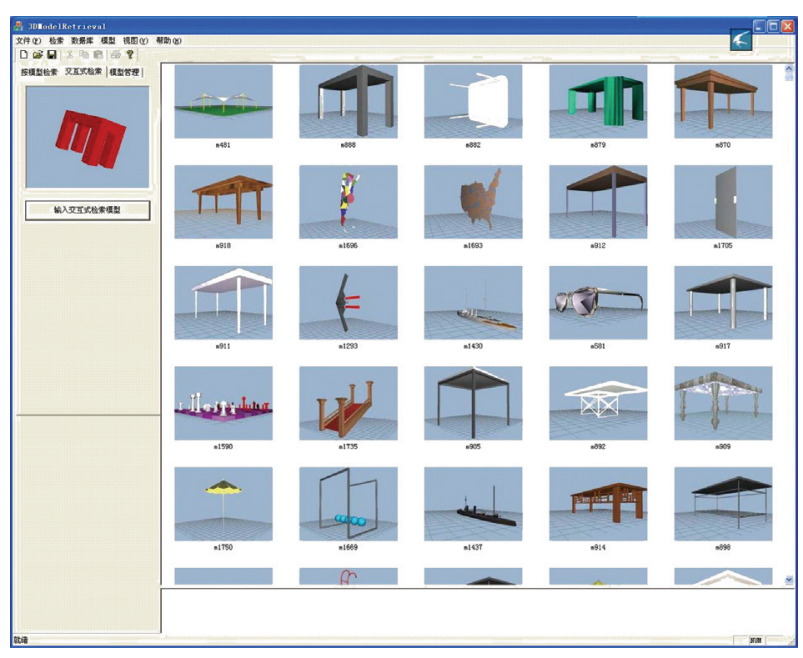

FIGURE 12: The new experimental result.

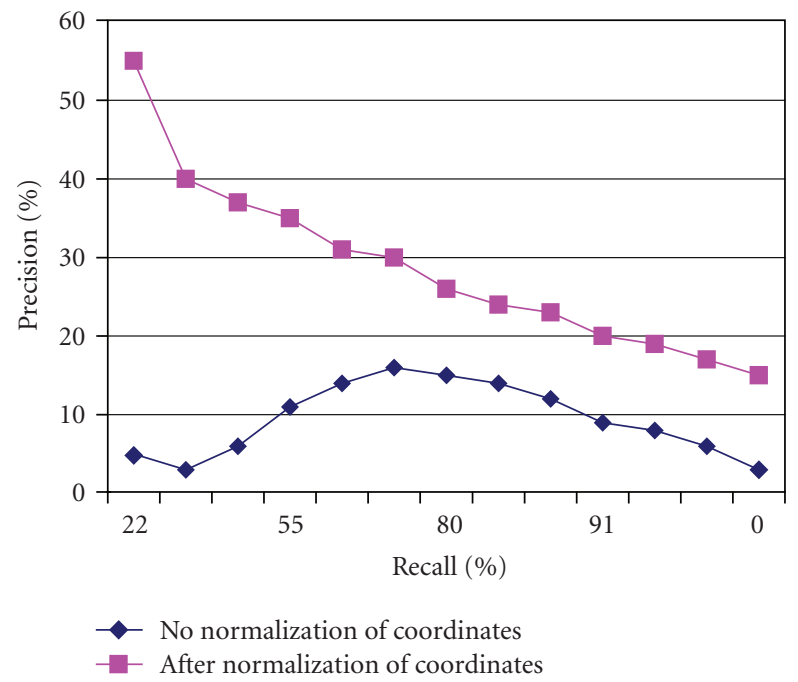

FIGURE 13: Precision recall of stool model.

rate (recall) and check rate (precision) to evaluate [17, 18]. The recall rate and the precision rate are as follows:

$$
\begin{aligned}
\text { Recall } & =\frac{\text { relevant correctly retrieved }}{\text { All relevant }}, \\
\text { Precise } & =\frac{\text { relevant correctly retrieved }}{\text { All retrieved }} .
\end{aligned}
$$

According to the retrieval results of plane model and stool model, we first calculate their recall rate and the precision rate and then draw precision-recall curve, as shown in Figure 11.

As can be seen from Figure 11, the plane model precision rate is far higher than that of the stool model. The precision rate of the stool is very low. How can we optimize the results?

\section{Retrieval Optimization}

In order to make retrieval results better, first of all, we use CPCA method $[19,20]$ to coordinate normalization of the pretreatment for the model. Retrieve again and the new result is shown in Figure 12.

Contrasting Figure 12 to Figure 10, we can see the retrieval result clearly improved.

To further contrast, we draw the precision-recall curve of the stool model before and after using the coordinate normalization, as shown in Figure 13.

As can be seen from Figure 13, the retrieval accuracy has greatly improved after coordinate normalization.

\section{Conclusion}

In the analysis and comparison of 3D model retrieval methods, we propose a new 3D model retrieval method with building blocks. Without hardware supporting, we construct query models by building blocks in the virtual environment.

Compared with the previous retrieval methods, it is very easy to construct query models. Users can easily express their intentions to query. The retrieval method we provided opens up a 3D model of the new ideas retrieval method, and it has a good retrieval performance at the same time.

\section{Acknowledgments}

This work is supported by the National Natural Science Foundation of China (no. 60736008). As the authors have acknowledged throughout the paper, the experiments conducted in their work rely heavily on the Princeton University 3D retrieval database PSB. The authors would also like to thank the anonymous reviewers.

\section{References}

[1] J. Wang, Y. He, H. Tian, and H. Cai, "Retrieving 3D CAD model by freehand sketches for design reuse," Advanced Engineering Informatics, vol. 22, no. 3, pp. 385-392, 2008.

[2] P. Min, J. A. Halderman, M. Kazhdan, and T. Funkhouser, "Early experiences with a 3D model search engine," in Proceedings of the 8th International Conference on 3D Web Technology, pp. 7-18, Saint Malo, France, March 2003.

[3] Y.-B. Yang, H. Lin, and Q. Zhu, "Content-based 3D model retrieval: a survey," Chinese Journal of Computers, vol. 27, no. 10, pp. 1297-1310, 2004

[4] R. Osada, T. Funkhouser, B. Chazelle, and D. Dobkin, "Shape distributions," ACM Transactions on Graphics, vol. 21, no. 4, pp. 807-832, 2002.

[5] T. Funkhouser, P. Min, M. Kazhdan, et al., "A search engine for 3D models," ACM Transactions on Graphics, vol. 22, no. 1, pp. 83-105, 2003.

[6] H. Sundar, D. Silver, N. Gagvani, and S. Dickinson, "Skeleton based shape matching and retrieval," in Proceedings of the Shape Modeling International (SMI '03), pp. 130-139, Seoul, Korea, May 2003.

[7] C. Zhang and T. Chen, "Indexing and retrieval of 3D models aided by active learning," in Proceedings of the 9th ACM International Conference on Multimedia, vol. 9, pp. 615-616, Ottawa, Canada, September 2001. 
[8] W. Liu, Y. Uehara, Y. Liu, et al., "3DMIRACLES: 3D model retrieval and visualization engine," in Visualization and Data Analysis 2005, vol. 5669 of Proceedings of SPIE, pp. 250-261, San Jose, Calif, USA, January 2005.

[9] M. T. Suzuki, "A web-based retrieval system for 3D polygonal models," in Proceedings of the Joint 9th IFSA World Congress and 20th NAFIPS International Conference (NAFIPS '01), vol. 4, pp. 2271-2276, Vancouver, Canada, July 2001.

[10] J. Pu and K. Ramani, "On visual similarity based 2D drawing retrieval," Computer Aided Design, vol. 38, no. 3, pp. 249-259, 2006.

[11] L. Cao, J. Liu, and X. Tang, "3D object retrieval using $2 \mathrm{D}$ line drawing and graph based relevance reedback," in Proceedings of the 14th Annual ACM International Conference on Multimedia (MM '06), pp. 105-108, Santa Barbara, Calif, USA, October 2006.

[12] M. J. Fonseca, A. Ferreira, and J. A. Jorge, "Towards 3D modeling using sketches and retrieval," in Proceedings of the EUROGRAPHICS Workshop on Sketch-Based Interfaces and Modeling, pp. 127-136, Grenoble, France, August 2004.

[13] T. Igarashi, S. Matsuoka, and H. Tanaka, "Teddy: a sketching interface for 3D freeform design," in Proceedings of the 26th Annual Conference on Computer Graphics and Interactive Techniques, pp. 409-416, Los Angeles, Calif, USA, August 1999.

[14] S. Hou and K. Ramani, "Classifier combination for sketchbased 3D part retrieval," Computers and Graphics, vol. 31, no. 4, pp. 598-609, 2007.

[15] H. Siegl, M. Hanheide, S. Wrede, and A. Pinz, "An augmented reality human-computer interface for object localization in a cognitive vision system," Image and Vision Computing, vol. 25, no. 12, pp. 1895-1903, 2007.

[16] H. Ichida, Y. Itoh, Y. Kitamura, and F. Kishino, "Interactive retrieval of 3D shape models using physical objects," in Proceedings of the 12th Annual ACM International Conference on Multimedia, pp. 692-699, New York, NY, USA, October 2004.

[17] K. Michael, F. Thomas, and R. Szymon, "Rotation invariant spherical harmonic representation of 3D shape descriptors," in Proceedings of the Eurographics/ACM SIGGRAPH Symposium on Geometry Processing, pp. 156-164, Aachen, Germany, June 2003.

[18] D.-Y. Chen, X.-P. Tian, Y.-T. Shen, and M. Ouhyoung, "On visual similarity based 3D model retrieval," Computer Graphics Forum, vol. 22, no. 3, pp. 223-232, 2003.

[19] D. V. Vranić, D. Saupe, and J. Richter, "Tools for 3D-object retrieval: Karhunen-Loeve transform and spherical harmonics," in Proceedings of the 4th IEEE Workshop on Multimedia Signal Processing (MMSP '01), pp. 293-298, Cannes, France, October 2001.

[20] D. V. Vranic, 3D model retrieval, Ph.D. dissertation, Department of Computer Science, University of Leipzig, Leipzig, Germany, 2004. 

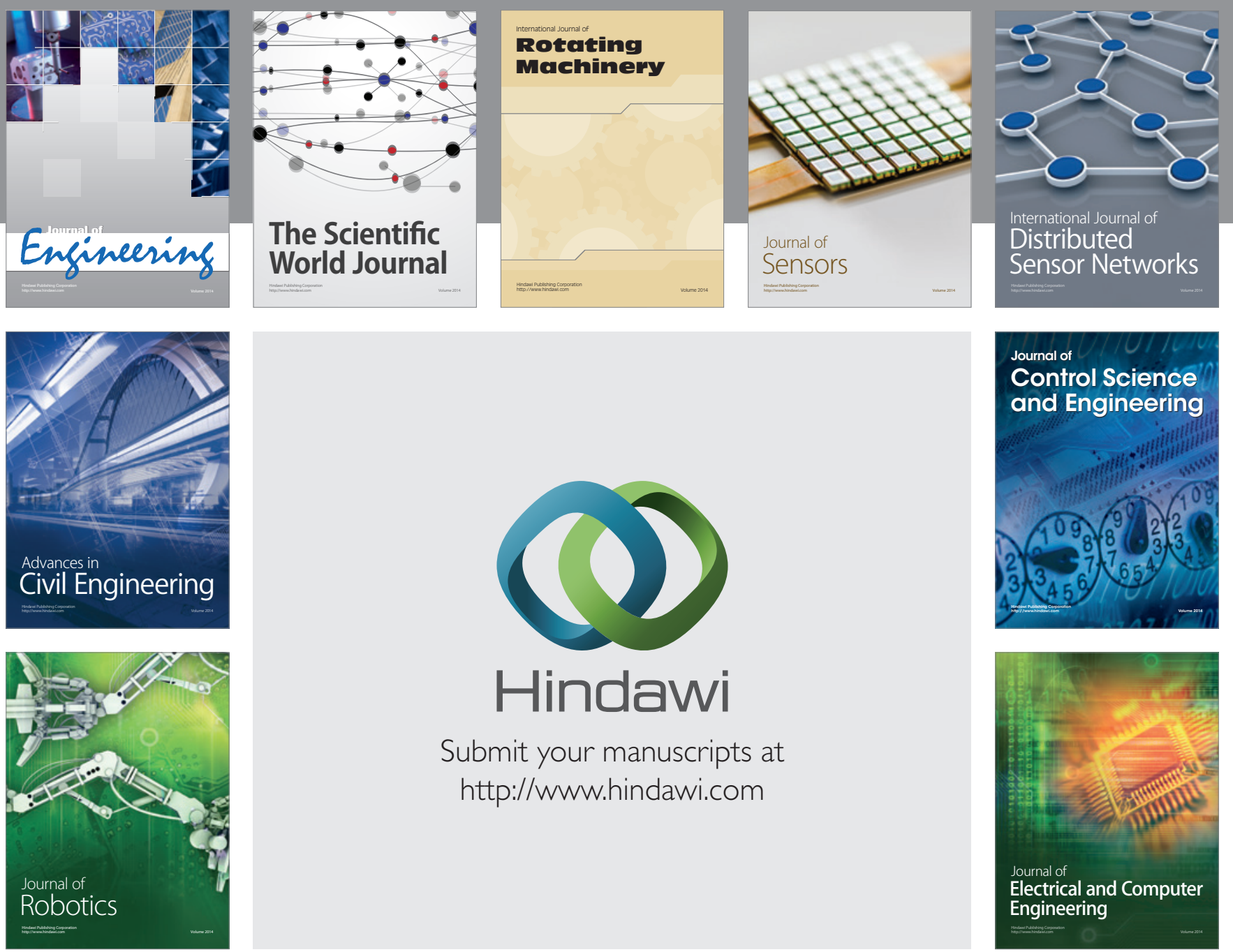

Submit your manuscripts at

http://www.hindawi.com
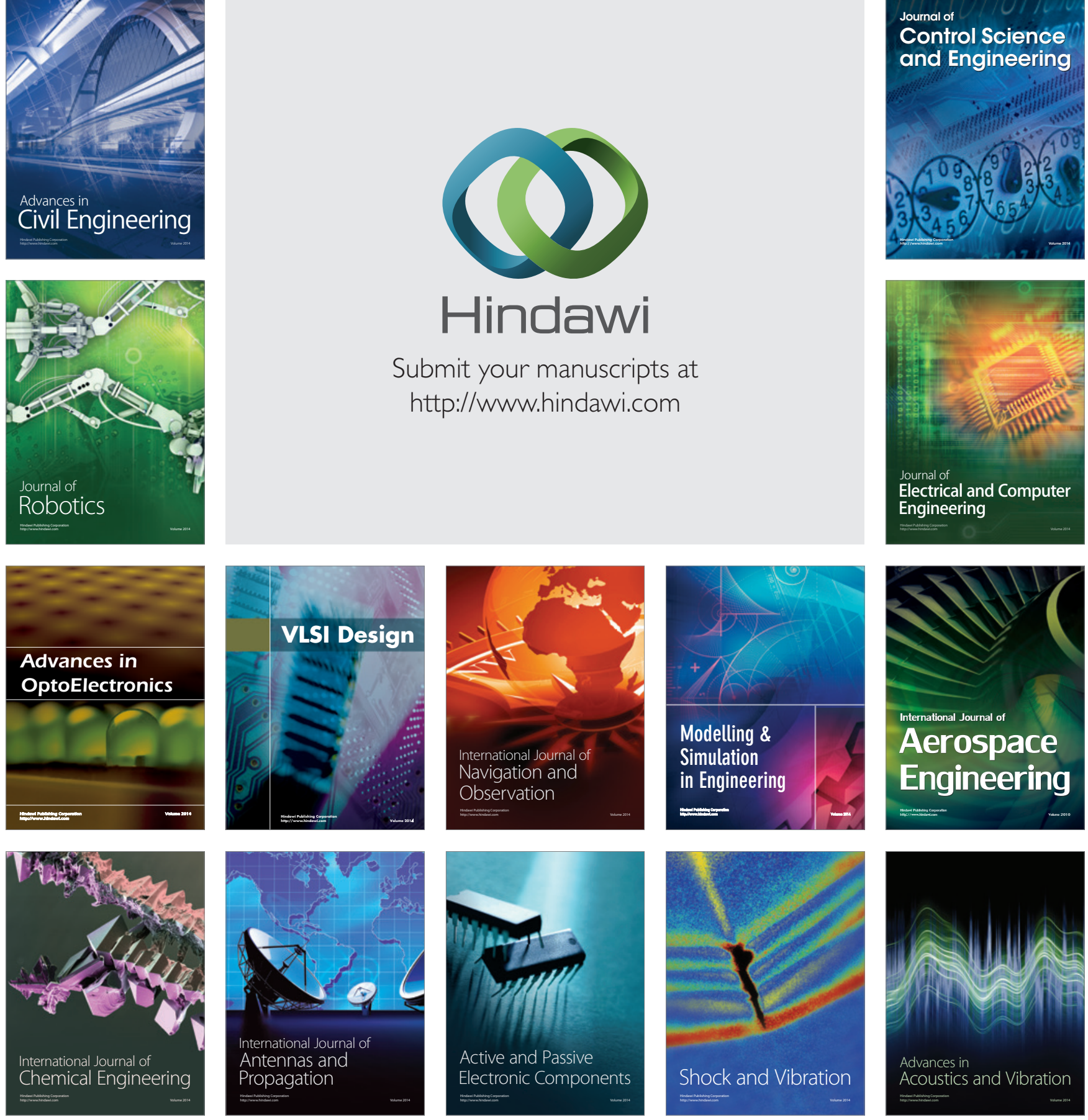